\title{
On certain expansions involving Bessel functions and Whittaker's $M$-functions
}

\author{
By S. C. Mitra.
}

(Received 2nd May, 1938. Read 6th May, 1938.)

(Received in revised form 29th August, 1938.)

1. Adopting the notation of Barnes ${ }^{1}$ and $\mathrm{Fox}^{2}$, let us write

$$
\begin{gathered}
\left.{ }_{p} f_{q}\left(a_{1}, a_{2}, \ldots a_{p} ; \rho_{1}, \rho_{2}, \ldots \rho_{q} ; x\right)=\sum_{r=0}^{\infty} \frac{\Gamma\left(a_{1}+r\right) \ldots \Gamma\left(a_{p}+r\right)}{\Gamma\left(\rho_{1}+r\right) \ldots \Gamma\left(\rho_{q}+r\right)} \frac{x^{r}}{\Gamma(r+1)}\right) \\
=\frac{\Gamma\left(a_{1}\right) \ldots \Gamma\left(a_{p}\right)}{\Gamma\left(\rho_{1}\right) \ldots \Gamma\left(\rho_{q}\right)}{ }_{p} F_{q}\left(a_{1}, a_{2}, \ldots a_{p} ; \rho_{1}, \rho_{2}, \ldots \rho_{q} ; x\right) .
\end{gathered}
$$

If $q>p-1$, the series on the right of (1) represents an integral function, while if $q=p-1$, the series converges only inside or on the circle $|x|=1$.

Now Barnes has proved that

$$
\begin{aligned}
& { }_{p} f_{q}\left(a_{1}, \alpha_{2}, \ldots a_{p} ; \rho_{1}+m, \rho_{2}, \ldots \rho_{q} ; x\right):= \\
& \quad \frac{1}{2 \pi i} \int_{C} \Gamma(-s) \frac{\Gamma\left(a_{1}+s\right) \ldots \Gamma\left(a_{p}+s\right)(-x)^{s}}{\Gamma\left(\rho_{1}+m+s\right) \Gamma\left(\rho_{2}+s\right) \ldots \Gamma\left(\rho_{q}+s\right)} d s,
\end{aligned}
$$

the integral being taken along a contour $C$, which encloses all the poles of $\Gamma(-s)$ but none of the other poles of the integrand. The contour $C$, as in Fox's paper, may be taken to be a rectangle except for necessary loops, having infinite sides parallel to the $x$-axis and sides of finite length parallel to the $y$-axis.

2. The following result is well-known ${ }^{3}$.

$$
{ }_{4} F_{3}\left(\begin{array}{c}
a, 1+\frac{1}{2} a, \quad c, \quad d ;-1 \\
\frac{1}{2} a, 1+a-c, 1+a-d
\end{array}\right)=\frac{\Gamma(1+a-c)}{\Gamma(1+a) \Gamma(1+a-d)} .
$$

In the above, let us put $a=\rho-1, c=-m$ and $d=-s$. We get

$$
{ }_{4} F_{3}\left(\begin{array}{c}
\rho-1, \frac{1}{2} \rho+\frac{1}{2},-m,-s ;-1 \\
\frac{1}{2} \rho-\frac{1}{2}, \rho+m, \rho+s
\end{array}\right)=\frac{\Gamma(\rho+m) \Gamma(\rho+s)}{\Gamma(\rho) \Gamma(\rho+m+s)} .
$$

${ }^{1}$ E. W. Barnes, Proc. London Math. Soc. (2), 5 (1906), 59-116.

$\because$ C. Fox, Proc. London Math. Soc. (2), 26 (1927), 201.

:W. N. Bailey, Generolised Hypergeometric Series (Cambridge Tract No. 32, 1935), 1. 28.

F. J. W. Whipple, Prot. London Math. Sor. (2), 25 (1926), $247-263$. 
Hence

$$
\begin{aligned}
& \frac{1}{\frac{1}{2}} \frac{\Gamma(-m) \Gamma(-s)}{\Gamma(\rho+m+s)}= \\
& \quad \sum_{r=0}^{\infty} \frac{(-1)^{r} \Gamma(\rho-1+r) \Gamma\left(\frac{1}{2} \rho+\frac{1}{2}+r\right)(\Gamma(r-m) \Gamma(r-s)}{\Gamma(r+1) \Gamma \frac{1}{\left(\frac{1}{2} \rho-\frac{1}{2}+r\right) \Gamma(\rho+m+r) \Gamma(\rho+s+r)}} .
\end{aligned}
$$

Combining this with (3), we get

$$
\begin{aligned}
& { }_{p} f_{q_{1}}\left(\alpha_{1}, \alpha_{2}, \ldots \alpha_{p} ; \rho_{1}+m, \rho_{2}, \ldots \rho_{q} ; x\right)= \\
& \frac{1}{2 \pi i} \frac{2}{\Gamma(-m)} \int_{C} \sum_{r=0}^{\infty} \frac{(-1)^{r} \Gamma\left(\rho_{1}-1+r\right) \Gamma\left(\frac{1}{2} \rho_{1}+\frac{1}{2}+r\right) \Gamma(r-m) \Gamma(r-s)}{\Gamma\left(\frac{1}{2} \rho_{1}-\frac{1}{2}+r\right) \Gamma\left(\rho_{1}+m+r\right) \Gamma\left(\rho_{1}+s+r\right) \Gamma(r+1)} \times \\
& \frac{\Gamma\left(\alpha_{1}+s\right) \ldots \Gamma\left(\alpha_{p}+s\right)}{\Gamma\left(\rho_{2}+s\right) \ldots \Gamma\left(\rho_{q}+s\right)}(-x)^{s} d s .
\end{aligned}
$$

The right hand side can be proved without difficulty to be equal to

$$
\begin{aligned}
\left.\frac{2}{\Gamma(-m)}\right)_{r=0}^{\infty} \frac{\Gamma\left(\rho_{1}-1+r\right) \Gamma\left(\frac{1}{2} \rho_{1}+\frac{1}{2}+r\right) \Gamma(r-m)}{\Gamma(r+1) \Gamma\left(\frac{1}{2} \rho_{1}-\frac{1}{2}+r\right) \Gamma\left(\rho_{1}+m+r\right)} \times \\
x_{p}^{r} f_{q}\left(\begin{array}{c}
\alpha_{1}+r, \ldots \ldots \alpha_{p}+r \\
\rho_{1}+2 r, \rho_{2}+r, \ldots \rho_{q}+r
\end{array}\right) . x
\end{aligned}
$$

It is easily seen that

$$
\sum_{r=0}^{\infty}(-1)^{r} \frac{\Gamma\left(\rho_{1}-1+r\right) \Gamma\left(\frac{1}{2} \rho_{1}+\frac{1}{2}+r\right) \Gamma(r-m) \Gamma(r-s)}{\Gamma(r+1) \Gamma\left(\frac{1}{2} \rho_{1}-\frac{1}{2}+r\right) \Gamma\left(\rho_{1}+m+r\right) \Gamma\left(\rho_{1}+s+r\right)}
$$

is uniformly convergent with regard to $s$, provided that

$$
R\left(\rho_{1}+2 m+2 s+1\right)>0 .
$$

Again

$$
\Gamma\left(\alpha_{1}+s\right) \sim \exp \left\{\left(a_{1}+s-\frac{1}{2}\right) \log s-s+\frac{1}{2} \log 2 \pi+O\left(\frac{1}{s}\right)\right\},
$$

when $|\arg s|<\pi$. As $s \rightarrow \infty$ along $C, I(s)$ remains finite. We have

$$
\begin{aligned}
& \frac{\Gamma(r-s) \Gamma\left(\alpha_{1}+s\right) \ldots \Gamma\left(a_{p}+s\right)(-x)^{s}}{\Gamma\left(\rho_{1}+s+r\right) \Gamma\left(\rho_{2}+s\right) \ldots \Gamma\left(\rho_{q}+s\right)} \sim \\
& \quad \exp \{(q-p+1)(s-s \log s)+s \log x+O(\log s)\} .
\end{aligned}
$$

If $p<q+1$ or $p=q+1$ and $\mid x_{1}<1$, the integrand tends to zero with exponential rapidity as $R(s) \rightarrow+\infty$. It follows that the order of integration and summation may be interchanged even when the range of integration is infinite . $^{1}$

\footnotetext{
${ }^{1}$ I am indebted to a referee for this suggestion.
} 
3. In (8) let us put $p=1, q=2, \rho_{1}=2 \alpha$ and $\rho_{2}=a+\frac{1}{2}$. Then ${ }_{1} F_{2}\left(\alpha ; 2 \alpha+m, \alpha+\frac{1}{2} ; x\right)=$ $\frac{2}{\Gamma(\alpha) \Gamma(-m)} \sum_{r=0}^{\infty} \frac{\Gamma(a+r) \Gamma(2 a-1+r) \Gamma(r-m)}{\Gamma(r+1) \Gamma\left(\alpha-\frac{1}{2}+r\right) \Gamma(2 a+m+r) \Gamma(2 a+2 r)} x^{r} \times$ ${ }_{1} F_{2}\left(a+r ; a+\frac{1}{2}+r, 2 \alpha+2 r ; x\right)$.

Writing $-x^{2}$ for $x$ and taking $m$ to be a positive integer, we get ${ }^{1}$ $x^{2 \alpha-1}{ }_{1} F_{2}\left(\alpha ; \alpha+\frac{1}{2}, 2 \alpha+m ;-x^{2}\right)=$ $2 \sqrt{\pi} \frac{\Gamma\left(\alpha+\frac{1}{2}\right) \Gamma(2 \alpha+m)}{\Gamma(\alpha)} \sum_{r=0}^{m}\left(\begin{array}{c}m \\ r\end{array}\right) \frac{\Gamma(2 \alpha-1+r)}{\Gamma\left(\alpha-\frac{1}{2}+r\right)} \frac{\Gamma\left(\alpha+\frac{1}{2}+r\right)}{\Gamma(2 \alpha+m+r)} J_{a-\frac{1}{2}+r}^{2}(x)$.

We can also easily deduce the following results

$$
\begin{aligned}
& x^{-2 \rho-2 m} J_{\rho+m}^{2}(x)= \\
& \frac{1}{\sqrt{ } \pi} \sum_{r=0}^{2 m}\left(\begin{array}{c}
2 m \\
r
\end{array}\right) \frac{\Gamma(2 \rho+r) \Gamma\left(\rho+m+\frac{1}{2}+r\right)}{\Gamma(\rho+m+1+r) \Gamma(2 \rho+2 m+1+r) \Gamma(2 \rho+2 r)} x^{2 r} \times \\
& { }_{1} F_{2}\left(\rho+m+\frac{1}{2}+r ; \rho+m+1+r, 2 \rho+2 r+1 ;-x^{2}\right) \\
& \frac{{ }_{1} F_{1}(\alpha ; \rho+m ; x)=}{\Gamma(\rho+m)} \sum_{r=0}^{m}\left(\begin{array}{c}
m \\
r
\end{array}\right) \frac{\Gamma(\rho-1+r) \Gamma(\alpha+r)}{\Gamma(\rho+m+r) \Gamma(\rho-1+2 r)}(-x)_{1}^{r} F_{1}(\alpha+r ; \rho+2 r ; x)
\end{aligned}
$$

whence we get, on writing $2 \rho+1$ for $\rho, 2 m$ for $m$ and $\alpha+\frac{1}{2}$ for $\alpha$,

$$
\begin{aligned}
& x^{-m} M_{\rho+m-a, \rho+m}(x)= \\
& \frac{\Gamma(2 \rho+2 m+1)}{\Gamma\left(\alpha+\frac{1}{2}\right)} \sum_{r=0}^{2 m}(-1)^{r}\left(\begin{array}{c}
2 m \\
r
\end{array}\right) \frac{\Gamma\left(\alpha+\frac{1}{2}+r\right) \Gamma(2 \rho+r)}{\Gamma(2 \rho+2 m+1+r) \Gamma(2 \rho+2 r)} M_{\rho-\alpha, \rho+r}(x),(13)
\end{aligned}
$$

where ${ }^{2}$

$$
M_{k, m}(x)=e^{-\frac{1}{2} x} x^{m+\frac{1}{2}} F_{1}\left(m+\frac{1}{2}-k ; 2 m+1 ; x\right),
$$

$2 m$ not being a negative integer.

Since $^{3}$

and

$$
J_{n}(x)=\frac{x^{-\frac{1}{2}}}{2^{2 n+\frac{1}{2}} i^{n+\frac{1}{2}} \Gamma(n+1)} M_{0, n}(2 i x)
$$

$$
I_{n}(x)=i^{-"} J_{n}(i x),
$$

${ }^{3}\left\{J_{v}(x)\right\}^{2}=\{\Gamma(v+1)\}^{-2}\left(\frac{1}{2} x\right)^{2}{ }_{1} F_{2}\left(v+\frac{1}{2} ; v+1,2 v+1 ;-x^{2}\right)$.

2 E. T. Whittaker and G. N. Watson, Modern Analysis (Cambridge, 1920), p. 338.

3 E. T. Whittaker and G. N. Watson, Modern Analysis (Cambridge, 1920), p. 360. 
it easily follows from the above that

$x^{-m-\frac{1}{2}} M_{m, a+m}(x)=$

$2 \sqrt{\pi} \frac{\Gamma(2 \alpha+2 m+1)}{\Gamma\left(\alpha+\frac{1}{2}\right)} \sum_{r=0}^{2 m}(-1)^{r}\left(\begin{array}{c}2 m \\ r\end{array}\right) \frac{\Gamma(2 \alpha+r) \Gamma(\alpha+1+r)}{\Gamma(\alpha+r) \Gamma(2 \alpha+2 m+1+r)} I_{a+r}\left(\frac{1}{2} x\right)$.

Taking $a=\rho+m$, we find that

$x^{-m+\frac{1}{2}} I_{\rho+m}\left(\frac{1}{2} x\right)=$

$\frac{1}{\sqrt{\pi}} \sum_{r=0}^{2 m}(-1)^{r}\left(\begin{array}{c}2 m \\ r\end{array}\right) \frac{\Gamma(2 \rho+r) \Gamma\left(\rho+m+\frac{1}{2}+r\right)}{\Gamma(2 \rho+2 m+1+r) \Gamma(2 \rho+2 r)} M_{-m, \rho+r}(x)$.

4. In the formula (4) let us put $d=\frac{1}{2} a+\frac{1}{2}$. We get

$$
{ }_{3} F_{2}\left(\begin{array}{c}
a, 1+\frac{1}{2} a, \quad c ;-1 \\
\frac{1}{2} a, 1+a-c
\end{array}\right)=\frac{\Gamma\left(\frac{1}{2}+\frac{1}{2} a\right) \Gamma(1+a-c)}{\Gamma(1+a) \Gamma\left(\frac{1}{2}+\frac{1}{2} a-c\right)} .
$$

Let us put $c=-s, a+1=2 \rho_{1}$ and combine with (3). We get, on proceeding as in Art. 2,

$$
\begin{gathered}
{ }_{p} f_{q}\left(\alpha_{1}, \alpha_{2}, \ldots \alpha_{p} ; \rho_{1}, \rho_{2}, \ldots \rho_{q} ; x\right)=\frac{2}{\Gamma\left(\rho_{1}\right)} \sum_{r=0}^{\infty} \frac{\Gamma\left(2 \rho_{1}-1+r\right) \Gamma\left(\rho_{1}+\frac{1}{2}+r\right)}{\Gamma(r+1) \Gamma\left(\rho_{1}-\frac{1}{2}+r\right)} x^{r} \times \\
{ }_{p} f_{q}\left(\alpha_{1}+r, \alpha_{2}+r, \ldots \alpha_{p}+r ; 2 \rho_{1}+2 r, \rho_{2}+r, \ldots \rho_{q}+r ; x\right)
\end{gathered}
$$

Taking $p=1, q=2, \rho_{1}=\alpha$ and $\rho_{2}=\alpha+\frac{1}{2}$, we get after a little simplification ${ }^{1}$

$$
x^{\alpha-\frac{1}{2}} J_{a-\frac{1}{2}}(2 x)=\frac{2 \sqrt{\pi}}{\Gamma(\alpha)} \sum_{r=0}^{\infty}(-1)^{r} \frac{\Gamma(2 \alpha-1+r) \Gamma\left(\alpha+\frac{1}{2}+r\right)}{\Gamma(r+1) \Gamma\left(a-\frac{1}{2}+r\right)} J_{a-\frac{1}{2}+r}^{2}(x),
$$

where $a \geqq \frac{1}{2}$.

In a similar manner we can prove that

$$
x^{\frac{1}{2} \rho} M_{\frac{1}{2} \rho-\alpha, \frac{1}{2} \rho-\frac{1}{2}}(x)=\frac{1}{\Gamma(\alpha)} \sum_{r=0}^{\infty} \frac{\Gamma(2 \rho-1+r)}{\Gamma(r+1) \Gamma(a+r)} M_{\rho-a, \rho-\frac{1}{2}+r}(x) .
$$

5. Again in (4) let us write $c=-s, d=\alpha+s$. We get

${ }_{4} F_{3}\left(\begin{array}{c}a, 1+\frac{1}{2} a, \quad-s, \\ \frac{1}{2} a, 1+a+s, 1+a-a-s\end{array}\right)=\frac{\Gamma(1+a+s) \Gamma(1+a-a-s)}{\Gamma(1+a) \Gamma(1+a-a)}$.

\section{Hence}

$\frac{1}{2} \frac{\Gamma(-s) \Gamma(\alpha+s)}{\Gamma(1+a-a)}=\sum_{r=0}^{\infty} \frac{(-1)^{r} \Gamma(a+r) \Gamma\left(1+\frac{1}{2} a+r\right) \Gamma(r-s) \Gamma(a+s+r)}{\Gamma(r+1) \Gamma\left(\frac{1}{2} a+r\right) \Gamma(1+a+s+r) \Gamma(1+a-a-s+r)}$.

1 This is a special case of equation (12) with $Z=z, C_{\nu}=J_{\nu}, v=a-\frac{1}{2}$. See G. N. Watson, Bessel Functions (Cambridge, 1922), p. 366. 
Combining this with (3), we get

$$
\begin{aligned}
& { }_{p} f_{q}\left(a_{1}, a_{2}, \ldots \alpha_{p} ; \rho_{1}, \rho_{2}, \ldots \rho_{q} ; x\right)= \\
& \frac{1}{2 \pi i} 2 \Gamma\left(1+a-a_{1}\right) \sum_{r=0}^{\infty} \int_{C}(-1)^{r} \frac{\Gamma(a+r) \Gamma\left(\frac{1}{2} a+1+r\right)}{\Gamma(r+1) \Gamma\left(\frac{1}{2} a+r\right)} \times \\
& \frac{\Gamma\left(a_{2}+s\right) \ldots \Gamma\left(a_{p}+s\right) \Gamma(r-s) \Gamma\left(\alpha_{1}+s+r\right)(-x)^{s}}{\Gamma\left(\rho_{1}+s\right) \Gamma\left(\rho_{2}+s\right) \ldots \Gamma\left(\rho_{q}+s\right) \Gamma(a+1+s+r) \Gamma\left(a+1-\alpha_{1}-s+r\right)} d s, \\
& =\frac{2}{\Gamma\left(a_{1}-a\right)} \sum_{r=0}^{\infty} \frac{\Gamma(a+r) \Gamma\left(\frac{1}{2} a+1+r\right)}{\Gamma(r+1) \Gamma\left(\frac{1}{2} a+r\right)} x^{r} \times \\
& \qquad p+1 f_{q+1}\left(\begin{array}{l}
a_{1}-a, a_{1}+2 r, a_{2}+r, \ldots a_{p}+r, \\
a+1+2 r, \rho_{1}+r, \rho_{2}+r, \ldots \rho_{q}+r
\end{array} ;-x\right),
\end{aligned}
$$

the change in the order of summation and integration being justifiable.

Taking $p=2$ and $q=1$, we have, on putting $a=\alpha_{1}-1$, ${ }_{2} F_{1}\left(a_{1}, \alpha_{2} ; \rho_{1} ; x\right)=$ $\frac{\Gamma\left(\rho_{1}\right)}{\Gamma\left(\alpha_{1}\right) \Gamma\left(\alpha_{2}\right)} \sum_{r=0}^{\infty} \frac{\Gamma\left(a_{1}-1+r\right) \Gamma\left(\alpha_{2}+r\right) \Gamma\left(\alpha_{1}+2 r\right)}{\Gamma(r+1) \Gamma\left(\rho_{1}+r\right) \Gamma\left(a_{1}-1+2 r\right)} x_{2}^{r} F_{1}\left(1, \alpha_{2}+r ; \rho_{1}+r ;-x\right)$.

Putting $x=-1$ in the above and writing $a_{1}+1$ and $\rho_{1}+1$ for $\alpha_{1}$ and $\rho_{1}$ respectively, we have, if $R\left(\rho_{1}-\alpha_{1}-\alpha_{2}\right)>0$,

${ }_{3} F_{2}\left(\alpha_{1}, \frac{1}{2} \alpha_{1}+1, \alpha_{2} ; \frac{1}{2} \alpha_{1}, \rho_{1} ;-1\right)=\frac{\rho_{1}-\alpha_{2}}{\rho_{1}} F_{2} F_{1}\left(\alpha_{1}+1, \alpha_{2} ; \rho_{1}+1 ;-1\right)$.

Dacca, India. 